\title{
INHIBIDORES DE LA TIROSIN-QUINASA EN EL TRATAMIENTO DEL CÁNCER DE VEJIGA MÚSCULO-INFILTRANTE Y EL CÁNCER DE PRÓSTATA HORMONO- REFRACTARIO
}

\author{
Hervé Wallerand', Grégoire Robert', Jean-Christophe Bernhard', Alain Ravaud² y \\ Jean-Jacques Patard ${ }^{3}$.
}

'Servicio de Urología. Bordeaux University Hospital. Bordeaux 2 Victor Segalen University. Bordeaux. 2 Servicio de Oncología Médica. Bordeaux University Hospital. Bordeaux 2 Victor Segalen University. Bordeaux. ${ }^{3}$ Servicio de Urología. Rennes University Hospital. Rennes. France.

Resumen.- OBJETIVO: Se sabe que varias proteín-quinasas son activadas en las células tumorales e impulsan el crecimiento y progresión tumoral. En el carcinoma de células renales metastásico, los inhibidores de la tirosinquinasa (TKIs) han logrado importantes beneficios en progresión libre de enfermedad y supervivencia global. Los TKls pueden ser también considerados como una prometedora opción de tratamiento en tumores vesicales y prostáticos. Nuestro objetivo fue dar a conocer los

CORRESPONDENCIA

Prof. Jean-Jacques Patard

Rennes University Hospital

CHRU Pontchaillou

Department of Urology

Rue Henri Le Guillou

35033 Rennes Cedex, France

jean-jacques.patard@chu-rennes.fr

Aceptado para publicar: 21 de octubre 2009 artículos más relevantes publicados para confirmar el interés de la utilización de los TKI en el tratamiento de estos tumores.

MÉTODOS: Se realizó una busqueda sistemática en PubMed y se revisaron los artículos recuperados. Las palabras clave utilizadas fueron: inhibidor de la tirosin-quinasa, inhibidor de la protein-quinasa, cáncer de próstata hormono-refractario, cáncer de vejiga músculoinfiltrante. Las publicaciones más relevantes de ciencia básica y ensayos clínicos controlados y aleatorizados fueron resumidas y analizadas.

RESULTADOS: En cuanto al cáncer de vejiga, el tratamiento TKI es una de las estrategias terapéuticas más estudiadas en el campo de la terapia dirigida. De hecho, se ha sugerido que dirigiendo solamente TK y/o asociándola con quimioterapia citotóxica puede representar una opción prometedora para tratar el cáncer de vejiga localmente avanzado y/o metastásico. En cuanto al cáncer de próstata hormono-refractario (CPHR), los datos recogidos son aún confusos. Los estudios de ciencia básica encontraron una interesante expresión de receptores EGF y VEGF en las células tumorales confirmando la idea de que los TKI podrían resultar eficientes en el CPHR. Sin embargo la mayoría de estudios publicados de fase ll encontraron un débil efecto sobre los síntomas y la calidad de vida sin ninguna disminución en los niveles de PSA o en la supervivencia general.

CONCLUSIÓN: Los TKls todavía no han alcanzado en tumores vesicales o prostátivos una eficacia similar a lo que se ha obtenido en el carcinoma renal metastásico. Se necesitan más estudios para establecer el papel de ese enfoque en tumores no renales. 
Palabras clave: Inhibidor de la proteín-quinasa Inhibidor de la tirosin-quinasa. Cáncer de vejiga. Cáncer de vejiga músculo infilrante. Cáncer de próstata. Cáncer de próstata hormono refractario. Terapia adyuvante.

Summary.- OBJECTIVES: Various protein kinases are known to be activated in cancer cells and drive tumor growth and progression. In metastatic renal cell carcinoma tyrosine-kinase inhibitors (TKIs) have achieved significant progression-free and overall survival improvements. For bladder and prostate cancers TKIs may also be considered as a promising treatment option. Our aim was to report the most relevant published articles to support the interest of the use of TKIs in the treatment of bladder and prostate cancer.

METHOD: PubMed database and bibliographies of retrieved articles were reviewed. The key words used were tyrosine-kinase inhibitor, protein-kinase inhibitor, hormone refractory prostate cancer, muscle invasive bladder cancer. The most relevant publications from basic science and clinical randomized controlled studies were summarized and analyzed.

RESULTS: Regarding bladder cancer, TKI treatment is one of the most studied therapeutic strategies in the field of targeted therapy. Indeed, it has been suggested that targeting TK alone and/or in association with cytotoxic chemotherapy may represent a promising option for treating locally advanced and/or metastatic bladder cancer. Concerning hormone refractory prostate cancer (HRPC), collected data are still confusing. Basic science studies found an interesting expression of EGF and VEGF receptors on cancer cells supporting the idea that TKIs could be efficient in HRPC. Nonetheless most of published clinical phase II studies found a weak effect on symptoms and quality of life without any decrease in PSA levels or overall survival.

CONCLUSION: TKIs have not yet achieved in bladder and prostate cancers similar efficacy to what has been obtained in metastatic renal cell carcinoma. Further studies are needed to establish the place of such an approach in non renal tumors.

Keywords: Protein kinase inhibitor. Tyrosine kinase inhibitor. Bladder cancer. Muscle invasive bladder cancer. Prostate cancer. Hormone refractory prostate cancer. Adjuvant therapy.

\section{INTRODUCCIÓN}

Se sabe que varias proteínas quinasas se activan en las células tumorales y dirigen el crecimiento y progresión del tumor. Por lo tanto, el bloqueo de la tirosin-quinasa (TQ) representa un enfoque racional para el tratamiento antitumoral. Los receptores de la tirosin-quinasa (RTKs) producen una señal mitogénica a través de la activación del gen Ras, que es un oncogén frecuentemente activado por mutaciones puntuales en diversos tumores humanos, incluyendo vejiga y próstata. Los RTKs han sido identificados como proto-oncogenes y la proliferación de células tumorales se ha asociado con actividad no regulada de los oncogenes (1). Sin embargo, los RTKs en células tumorales pueden también activar PI-3K que conduce a la activación de Akt y señales anti-apoptóticas a la célula a través de la fosforilación de BAD. Ambas vías Ras-dependiente y Ras-independiente permiten que las células tumorales proliferen selectivamente lo que sugiere que la focalización hacia una vía única no sería suficiente para el tratamiento eficaz del cáncer (Figura 1). La investigación básica en la terapia dirigida sugiere cada vez más que la inhibición de los diferentes objetivos representa una opción de tratamiento prometedora.

Los fármacos con diana molecular específica se dividen en 3 grupos:

a) Fármacos que actúan sobre la superficie celular de los receptores,

b) Fármacos que actúan sobre las vías intracelulares, $y$

c) Medicamentos que actúan sobre los inhibidores del proteosoma.

Los inhibidores de la tirosin-quinasa (TKIs) actúan sobre la superficie celular de los receptores que se sabe es anormalmente activa en los tumores. Se han desarrollado y aprobado para uso clínico numerosos TKls: gefitinib, erlotinib, sunitinib, lapatinib, nilotinib y dasatinib. Se ha demostrado que reducen la proliferación celular en líneas celulares de tumores humanos y xenoinjertos y que también aumentan la apoptosis, inducen la detención del ciclo celular y disminuyen la angiogenesis (2). Recientemente ha surgido la idea de que dirigiéndose a varios TK sería más efectivo que limitándose a activar un único TK (3). Se ha demostrado que varios receptores de la tirosin-quinasa como el EGFR, PDGFR, IGFR, VEGFR, FGFR, y HGFR se sobreexpresan en tumores vesicales y/o de prostáticos. Entre estos, el EFGR y las vías de la angiogénesis son especialmente interesantes porque se dispone en la práctica clínica de una diana específica para los fármacos.

En la metástasis de carcinoma de células renales, los inhibidores de la tirosin-quinasa (TKIs) han logrado mejorar la progresión libre de enfermedad y la supervivencia global más que otros tratamientos (4). En los tumores de vejiga y próstata, los TKls tam- 
bién pueden ser considerados como una prometedora opción de tratamiento. Nuestro objetivo fue dar a conocer los artículos más relevantes publicados para apoyar el interés de la utilización de los TKls en el tratamiento de los tumores de vejiga y próstata.

\section{MATERIAL Y MÉTODOS}

Se revisaron sistemáticamente la base de datos PubMed y las bibliografías de los artículos recuperados. Las palabras clave utilizadas fueron: inhibidor de la tirosin-quinasa, inhibidor de la proteína-quinasa, cáncer de próstata hormono refractario, cáncer de vejiga músculo-infiltrante. Se resumieron y discutieron las publicaciones más relevantes de ciencias básica y ensayos clínicos controlados y aleatorizados. Los estudios clínicos con una metodología deficiente o sin grupo de control fueron sistemáticamente rechazados.

\section{TKI en cáncer de vejiga}

El cáncer de vejiga es la segunda neoplasia genito urinaria más común en todo el mundo. La incidencia del cáncer de vejiga está aumentando, y se esperaron casi 360.000 nuevos casos en 2008 junto con 145.000 muertes (5). El cáncer de vejiga representa una de los tumores humanos más costosos, cuando se consideran los protocolos de vigilancia utilizados en la actualidad y el hecho de que muchos pacientes viven durante largos períodos de tiempo después del diagnóstico, especialmente en el cáncer de vejiga no músculo-infiltrante (6). De todos los nuevos casos diagnosticados de este tumor, el $70 \%$ son no músculo infiltrante (CVNMI) (Ta, Tl, y Tis), pero tanto hasta el $50-70 \%$ recurrirán y el $10-20 \%$ de ellos evolucionan mediante invasión de la membrana basal (T2-T4). En la primera presentación, casi el 20\% de los tumores vesicales son infiltrantes (cáncer vesical músculo-infiltrante. CVMI). El desafío en los CVNMI

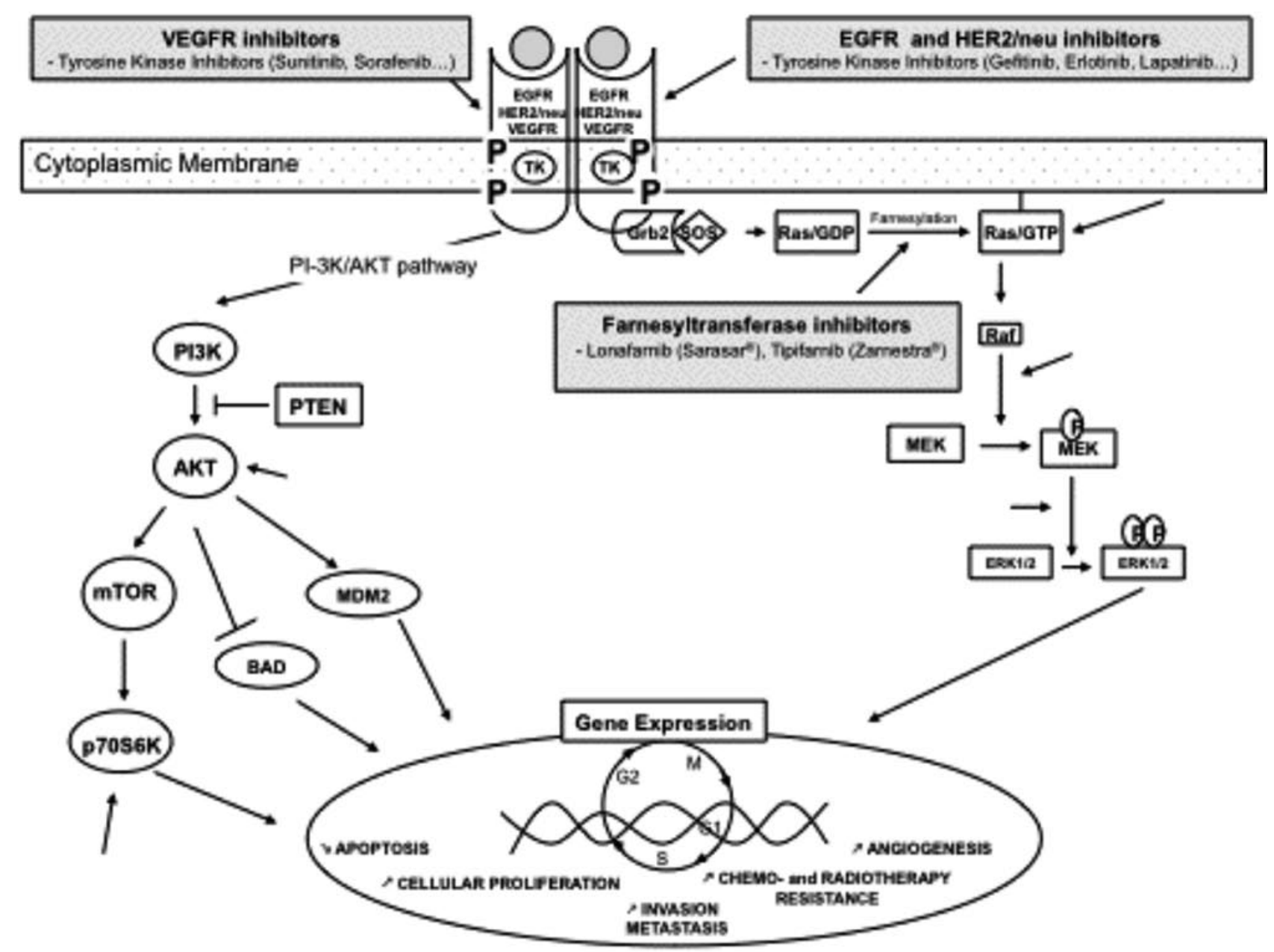

FIGURA 1. Terapias enfocadas a las vías de señalización implicadas en el tumor vesical y los inhibidores de la tirosin-quinasa (TKIs). 
sigue siendo predecir mediante el uso de biomarcadores moleculares qué tumores recurrirán y/o progresarán. Algunos de estos marcadores moleculares son también útiles en el tratamiento, ya que pueden ser utilizados como dianas, principalmente en el cáncer de vejiga localmente avanzado o metastásico. Los avances en la investigación molecular han conducido a una mejor comprensión de la biología tumoral del carcinoma urotelial y a desarrollar nuevas herramientas que permiten a los médicos seleccionar con más precisión el tratamiento óptimo para cada paciente. Las modalidades habituales de tratamiento de cáncer vesical (quimioterapia y radioterapia) siguen siendo decepcionantes debido a su toxicidad reforzando nuevamente la justificación para un tratamiento específico. El carcinoma urotelial está fuertemente asociado con mutaciones de las diversas vías moleculares implicadas en la proliferación celular, la angiogénesis tumoral y la apoptosis. Entre ellas, el receptor del factor de crecimiento epitelial (RFCE) y la angiogénesis tumoral son dianas importantes en la terapia dirigida a estas vías que, recientemente, estan disponibles en clínica.

\section{Focalización del RFCE (receptor del factor de creci- miento epitelial) en el cáncer de vejiga}

El RFCE es el transductor de señales implicado en la regulación de diversos procesos neoplásicos, tales como la progresión del ciclo celular, la inhibición de la apoptosis, la mobilidad de las células tumorales, invasión y metástasis (7). La activación del RFCE participa en la activación de la angiogénesis tumoral mediante la estimulación de la expresión del factor de crecimiento endotelial vascular (FCEV). La expresión aberrante del RFCE se ha demostrado en muchos tipos de cáncer, incluyendo el cáncer de vejiga y se ha asociado con la estadificación, el grado y la supervivencia global $(2,7)$.

También se ha asociado con la resistencia a la quimioterapia citotóxica común en varios tumores de nuestra econonomía, incluyendo el cáncer de vejiga (8). En el análisis multivariante, la presencia de expresión de RFCE en el cáncer vesical ha demostrado ser un predictor independiente de enfermedad invasiva, progresión en la estadificación y escasa supervivencia (9). La immunopositividad del RFCE en el tumor vesical se ha evaluado en estudios, que informaron de tasas de expresión del 23\% a $100 \%$ (10). La desregulación del RFCE en las neoplasias puede producirse por diferentes mecanismos como la sobreexpresión del receptor, mutaciones, alteraciones en el proceso de dimerización, y activación de bucles autocrinos para factor de crecimiento. El nivel de expresión de proteína RFCE no está por lo general considerado como un marcador biológico fiable de actividad de los TKI anti-RFCE (10). Se ha demostrado que los inhibidores del RFCE tienen efectos antiproliferativos y antiangiogénicos en modelos preclínicos (11). En esta clase, el gefitinib, lapatinib, y erlotinib son actualmente aceptados para uso clínico en varios tumores humanos y están bajo evaluación en el cáncer de vejiga (Tabla I).

El gefitinib conduce a la detención del ciclo celular en fase G1, implicando el incremento en la expresión del, inhibidor de la p27 $7^{\text {Kipl }}$ quinasa dependiente de ciclina (CDK) y en la disminución de la expresión de la CDK2-4-6, ciclina D1 y D3. Se observaron efectos similares después de tratamiento con erlotinib (11).

El lapatinib tiene como objetivo tanto el RFCE como el HER2/neu en contraste con el gefitinib, que es únicamente un inhibidor de RFCE. El lapatinib reduce la viabilidad celular en forma dosis-dependiente en líneas celulares de cáncer vesical RT112 y J82 (12). Asimismo se identificó un efecto sinérgico antitumoral de gefitinib y lapatinib con agentes citotóxicos en modelos preclínicos, y el lapatinib y los fármacos citotóxicos que se utilizan actualmente en el manejo del cáncer de vejiga metastásico mostraron sinergia dependiente de programación. Sin embargo, se han observado discrepancias entre la expresión de RFCE y la respuesta al RFCE -TKls en el cáncer de pulmón de células no microcítico.

Un enfoque alternativo podría consistir en explorar los agentes del flujo descendente de la vías de señalización del RFCE como STAT 5A y 5B, y cateninas- $\gamma$ (transductores de señal y activadores de transcripción) (13). Por otra parte, se ha demostrado que algunos genes como el GRG1 puede conferir resistencia al RFCE-TKI. El GRG1 está regulado al alza en tumores resistentes a gefitinib, lo que lleva a la hipótesis de que tomar como objetivo el GRG 1 podría ser una opción prometedora en tumores impulsados por RFCE. Por último, recientemente se ha demostrado que la sensibilidad al RFCE-TKI requiere expresión de E-cadherina en las células de carcinoma urotelial (14). Lamentablemente no se ha demostrado que el gefitinib asociado con Gemcitabina/cisplatino (GC) mejore la supervivencia en comparación con el GC o el MVAC sólo (15).

\section{Focalización de la HER2/neu en el cáncer de vejiga}

Se ha demostrado que la proteína HER2/neu (ErbB2) se expresa en el $2 \%$ al $74 \%$ de los tumores vesicales 10 y varios estudios han sugerido que la positividad de la HER2/nev está asociada con la pro- 


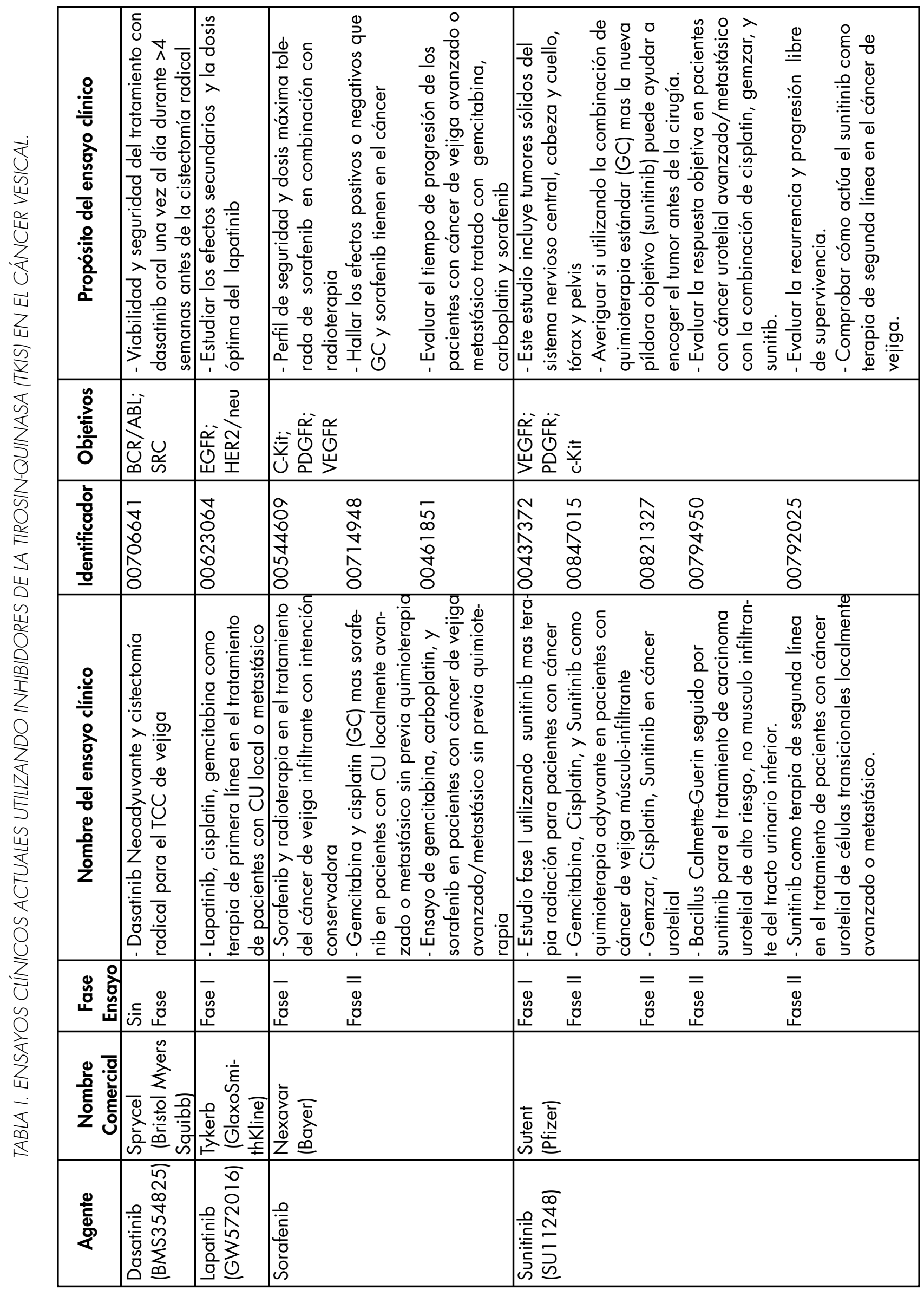




\begin{tabular}{|c|c|c|c|c|c|c|c|}
\hline 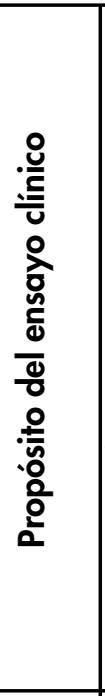 & 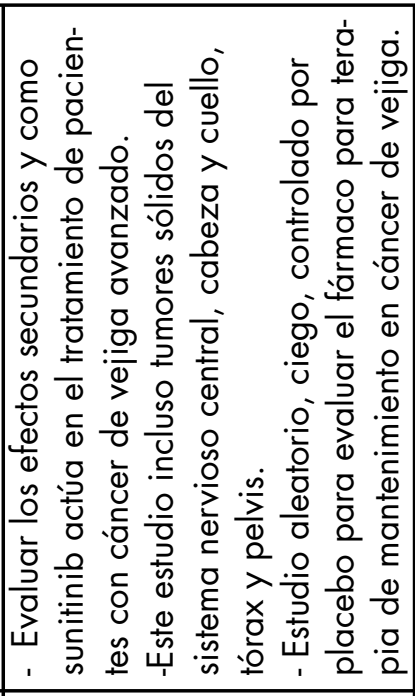 & 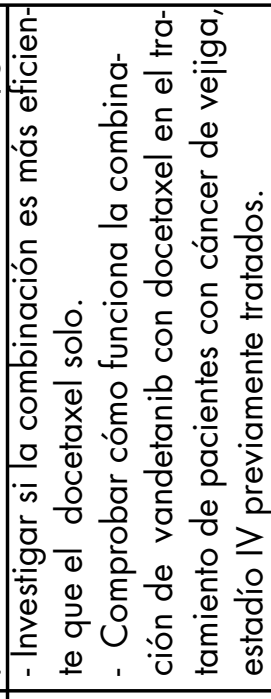 & 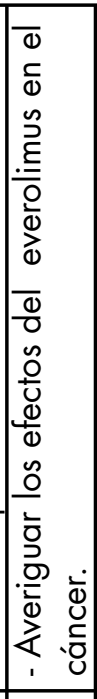 & 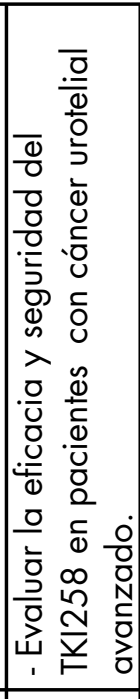 & 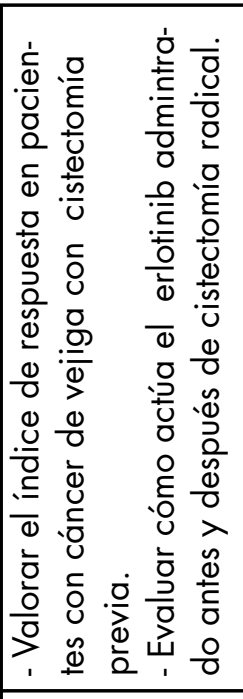 & 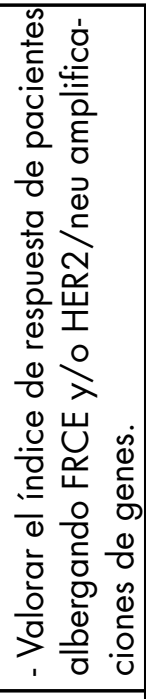 & 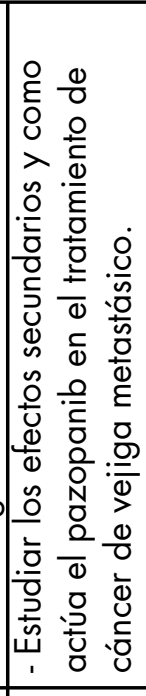 \\
\hline 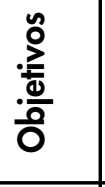 & & 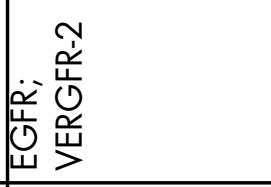 & 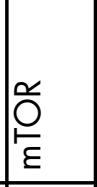 & 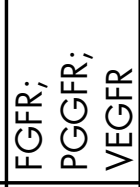 & \begin{tabular}{|l} 
嵌 \\
岀 \\
\end{tabular} & 쑸 & 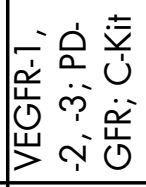 \\
\hline 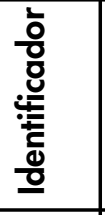 & 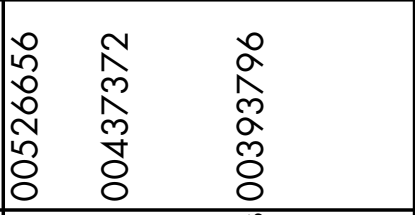 & 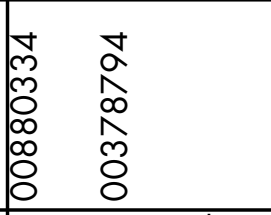 & 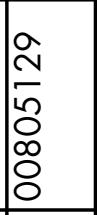 & 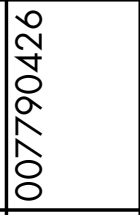 & 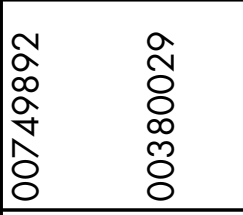 & 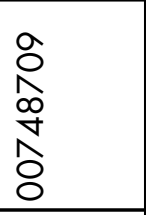 & 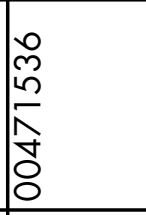 \\
\hline 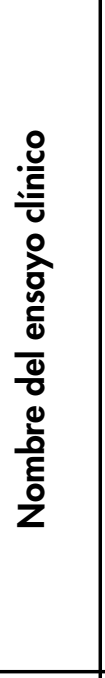 & 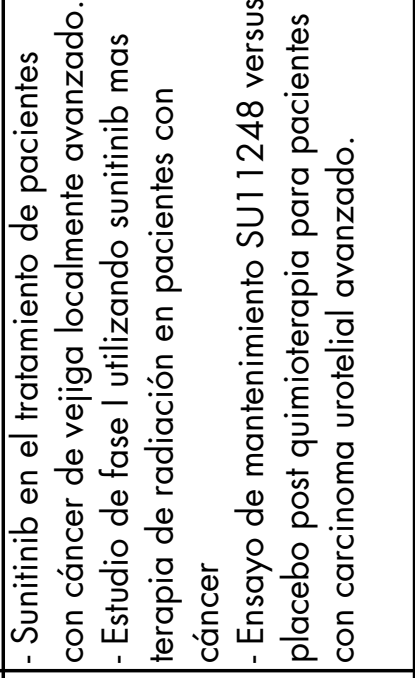 & 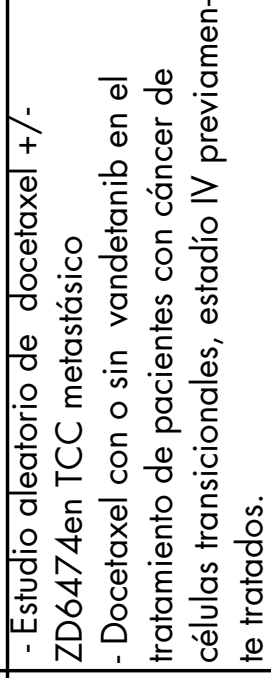 & 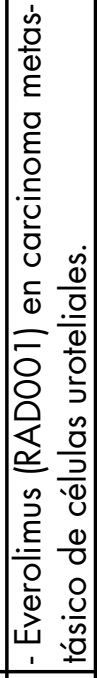 & 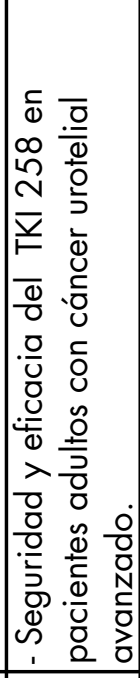 & 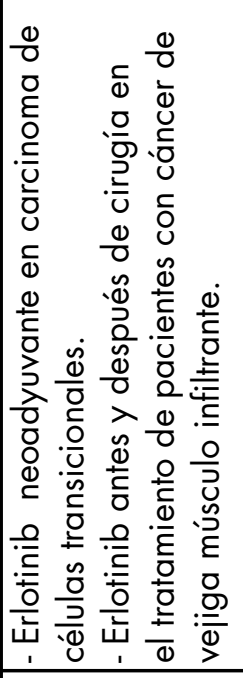 & 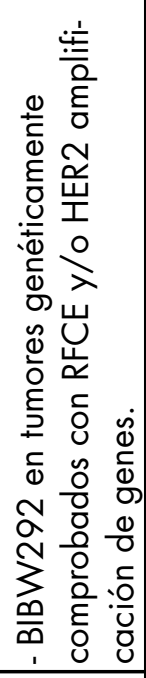 & 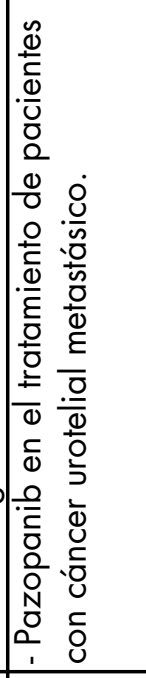 \\
\hline 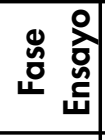 & 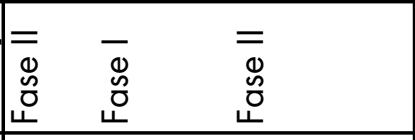 & $\begin{array}{|ll|} & = \\
\infty & 0 \\
心 & 0 \\
0 & 0 \\
\end{array}$ & 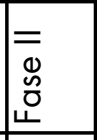 & \begin{tabular}{|l}
$=$ \\
$\infty$ \\
0 \\
$\dot{\sim}$ \\
\end{tabular} & 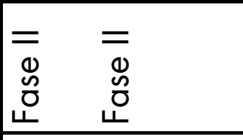 & 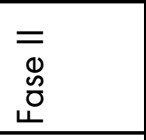 & \begin{tabular}{|l}
$=$ \\
$\infty$ \\
$\ddot{\infty}$ \\
\\
\end{tabular} \\
\hline 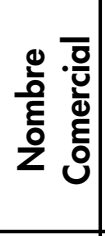 & & 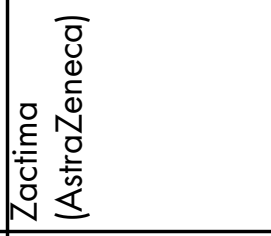 & 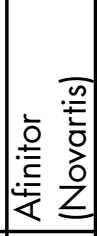 & 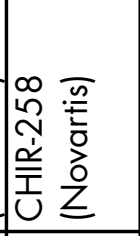 & 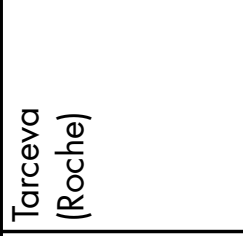 & 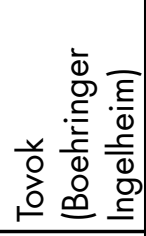 & 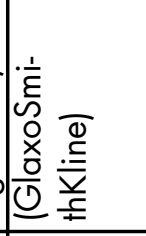 \\
\hline 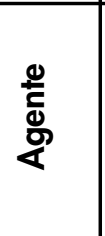 & & 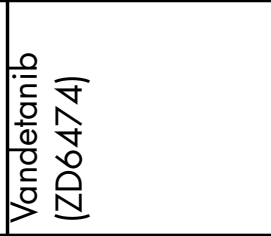 & 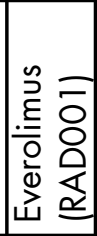 & $\begin{array}{l}\infty \\
\infty \\
\sim \\
\bar{v} \\
\bar{F}\end{array}$ & 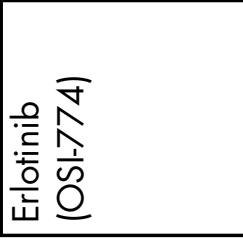 & 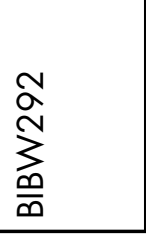 & 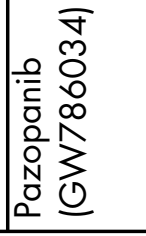 \\
\hline
\end{tabular}


gresión del tumor $(16,17)$. Sin embargo, el pronóstico del valor de la expresión HER2/neu permanece controvertido, ya que en 184 pacientes con CVNMI y CVMI, la expresión de HER2/neu no se encontró asociada con el estadio, grado, o supervivencia (9). La expresión combinada de RFCE y HER2/neu está presente en el $34 \%$ de los tumores, proporcionando así un razonamiento de base para la inhibición de RFCE/HER2/neu (18). El lapatinib es un TKI tanto de RFCE como de HER2/neu, que ha mostrado un beneficio moderado en un estudio fase II en neoadyuvancia (19).

Se ha informado que la inmuno-reactividad ErbB-2 y erbB-3 en los tumores vesicales va del $20 \%$ a $56 \%$ y $11 \%$ a $30 \%$, respectivamente (10). Por último, la co-expresión ErbB 1-2 y ErbB2-3 ha sido reconocido como un factor pronóstico independiente de baja supervivencia a largo plazo (17).

\section{Diana RFCVE (receptor del factor de crecimiento vas- cular endotelial) en el cáncer de vejiga}

La angiogénesis tiene un papel crítico en la progresión y en la metástasis del cáncer de vejiga (20). Se ha averiguado que la expresión del gen FCVE está relacionada con la supervivencia cáncerespecífica en pacientes con tumores vesicales localmente avanzados (21). La angiogénesis también pueden ser cuantificada por la densidad microvascular (DMV), que predice la posterior invasión muscular en CVNMI (22) y se correlaciona con la estadificación tumoral, grado y pobres resultados en el cáncer de vejiga (23).
Los TKIs dirigidos al FCVE incluyen sunitinib y sorafenib, que están bajo evaluación como TKls multi-diana en el cáncer vesical (Tabla I). El sunitinib y sorafenib son TKls que no sólo inducen a la inhibición de la angiogénesis, sino también a la activación de la apoptosis. El sunitinib ha demostrado actividad, tanto como agente único como en combinación con cisplatino en las líneas de células de cáncer de vejiga (24). Sobre la base del efecto de supervivencia libre de progresión del sunitinib y sorafenib en pacientes con carcinoma renal metastásico y en el papel potencial de la angiogénesis tumoral en la progresión del cáncer de vejiga, estos agentes han sido probados en cáncer de vejiga y mostraron actividad antitumoral contra el carcinoma urotelial humano como agente único y en combinación con cisplatino (25).

\section{Otros TKls en el cáncer de vejiga}

Varias vías moleculares que están implicadas en la carcinogénesis y progresión de los tumores vesicales podrían ser atacadas por agentes tales como los inhibidores de la histona deacetilasa, los inhibidores del proteosoma y los inhibidores de la farnesiltransferasa. TKIs han demostrado ser eficientes en la orientación de la farnesil transferasa (Ftasa), que es responsable de una modificación post traslaciónal (farnesilación) necesaria para las proteínas implicadas en las vías de transducción de señal, tales como los inhibidores de las proteínas Ras proteins (26). Los inhibidores de la Ftasa incluyen los TKls tipifarnib y ionofarnib que sólo mostraron respuestas pobres en los estudios de fase II $(27,28)$.

\section{TABLA II. EXPRESIÓN RFCE EN CÉLULAS PROSTÁTICAS DEPENDIENDO DE LA EXPOSICIÓN A TERAPIA ANTI-ANDROGÉNICA Y/O PROGRESIÓN TUMORAL DEL CÁNCER.}

\begin{tabular}{|l|c|c|}
\hline & Receptor & Expresión del receptor en células prostáticas \\
\hline $\begin{array}{l}\text { Di Lorenzo et al. (37) } \\
\text { 2002, Clin Cancer Res }\end{array}$ & ErbB-1 & $\begin{array}{c}41 \% \text { en CaP sin tratar hormonalmente } \\
76 \% \text { en CaP tratado con anti-andrógenos } \\
100 \% \text { en CPHR }\end{array}$ \\
\hline $\begin{array}{l}\text { Shah et al. (38) } \\
\text { 2006, Prostate }\end{array}$ & ErbB-1 & $\begin{array}{r}\text { OR }=6.67 \text { en PCHR } \\
\text { (comparado con el CaP sin tratar hormonalmente) }\end{array}$ \\
\hline Osman et al. (39) & ErbB-2 & $\begin{array}{r}20 \% \text { en CaP sin tratar hormonalmente } \\
67 \% \text { en CaP tratado con anti-andrógenos } \\
80 \% \text { en CaP metastásico }\end{array}$ \\
\hline
\end{tabular}

CaP: Cáncer de próstata; CPHR: Cáncer de próstata hormono-refractario 


\section{Los TKI en el cáncer de próstata}

El cáncer de próstata (CaP) es la segunda causa de mortalidad por cáncer entre los hombres de Europa occidental y de los Estados Unidos (5). La mayoría de muertes están causadas por metástasis de cáncer de próstata hormono-refractario (CPHR). El CaP avanzado o metastásico tratado primariamente con terapia de deprivación androgénica, ya sea por castración quirúrgica (orquiectomía) o castración médica con antagonistas de la hormona liberadora luteinizante (LHRH) con o sin anti-andrógenos (29). Aunque estos tratamientos dan como resultado la estabilización o regresión de la enfermedad metastásica en el $80 \%$ de los pacientes (30), la mayoría de estos pacientes progresan a CPHR (31). La progresión a CPHR resulta en que menos del $50 \%$ de los pacientes viven a los 5 años. El tratamiento de primera línea para el CPHR es docetaxel en combinación con prednisona $(32,33)$. La quimioterapia basada en sesiones de docetaxel cada 3 semanas demostró una importante mejoría en la calidad de vida y en la supervivencia global en comparación con el mitoxantrone. Sin embargo, el promedio de beneficio de supervivencia sigue siendo decepcionantemente corto (2 a 3 meses) y una proporción significativa de los pacientes no responden a la quimioterapia 32 , 33). Para los pacientes con enfermedad refractaria al docetaxel, no hay tratamiento de segunda línea con efectividad probada y se necesitan nuevas dianas celulares para el tratamiento.

Diversas moléculas implicadas en la proliferación celular, apoptosis y angiogénesis pueden ofrecer enfoques alternativos para el tratamiento del CPHR. El RFCE y RFCVE activan dos de las principales vías de transducción de señales que conducen a la progresión o metástasis tumoral. Los inhibidores de la tirosin-quinasa (TKIs) están interfiriendo con estos receptores, por lo tanto representan un enfoque prometedor para el tratamiento del CPHR.

\section{Actividades del RFCE y el FRGVE en el cáncer de prós- tata (Tabla II)}

La familia de receptores del factor epidérmico de crecimiento incluyen los receptores RFCE o ErbB-1, Su 2/neu o erbB-2, Her 3 o erbB-3 y 4 o Su ErbB4. Cuando se estimulan, el RFCE y el erbB-2 puede activar las vías de transducción MAP quinasa y $\mathrm{PI} 3$ quinasa que han demostrado estar involucradas en la progresión del CaP y en la diferenciación a CPHR (34, 35). La activación de la vía de señalización del RFCE desempeña un papel importante en el crecimiento, proliferación y supervivencia de muchas tumores sólidos (36). En el CaP, la expresión de RFCE parece estar asociada con la progresión de la enfermedad y se ha estudiado la densidad micro vascular como factor pronóstico de la agresividad del cáncer de próstata.

Di Lorenzo et al, encontraron que la expresión ErbB-1 aumenta en el CaP, y hace que éste se vuelva mas agresivo (37). Compararon la expresión inmunohistoquímica del ErbB-1 en las diferentes etapas mismo. La expresión ErbB-1 fue aumentando progresivamente desde el $41 \%$ en $\mathrm{CaP}$ sin tratamiento, hasta el $76 \%$ en $\mathrm{CaP}$ tratados con anti-andrógenos, y finalmente al $100 \%$ en el CPHR. Del mismo modo Shah et al, descubrieron que la expresión de ErbB1 está fuertemente asociada con el estadio hormono-refractario (cociente de probabilidades $=6,67$, $p<0,001)(38)$.

Tambien se ha demostrado que ErbB-2 está sobre-expresado en el CPHR. Osman et al, estudiaron la expresión ErbB-2 en 45 tumores primarios sin tratar hormonalmente, en 34 tumores primarios después de la terapia androgénica, y en 20 lesiones metastásicas (39). Encontraron una sobre-expresión de ErbB-2 en un $20 \%, 67 \%$ y $80 \%$ de los pacientes, que sugiere que la expresión ErbB-2 aumenta con la agresividad de la enfermedad. Por último, se ha demostrado un cambio regulación paracrina a regulación autocrina del RFCE en el CPHR (40).

La angiogénesis es también un paso importante en la progresión del cáncer de próstata temprano a enfermedad avanzada y es esencial en la metástasis de los tumores sólidos (41).

\section{Diana RFCE en el cáncer de próstata}

El uso de la terapia dirigida mediante TKls a las vía de señalización del RFCE ha mostrado una inhibición del crecimiento tumoral no solo en los CaP andrógeno- dependientes, sino también en CPHR (42). Se han investigado varios fármacos TKI, pero sólo unos pocos han alcanzado la fase II o III de ensayo clínico.

El gefitinib (Iressa®, de AstraZeneca) se dirige a ErbB-1 y es el TKI más ampliamente estudiado en el CaP. Ha mostrado actividad antitumoral en estudios preclínicos con una inhibición de crecimiento de 70 a $80 \%$ de las líneas célulares de xenoinjertos de tumores de próstata humanos transfectados a ratones (43). Ha pasado por numerosos estudios de monoterapia en fase I con una amplia gama de tumores sólidos, incluyendo CaP (44-46). De los 19 pacientes con CPHR inscritos en estos estudios de fase I, uno tuvo una respuesta en el tejido objetivo con disminución $>50 \%$ en el nivel de PSA durante 6,5 meses, otro tuvo disminución 


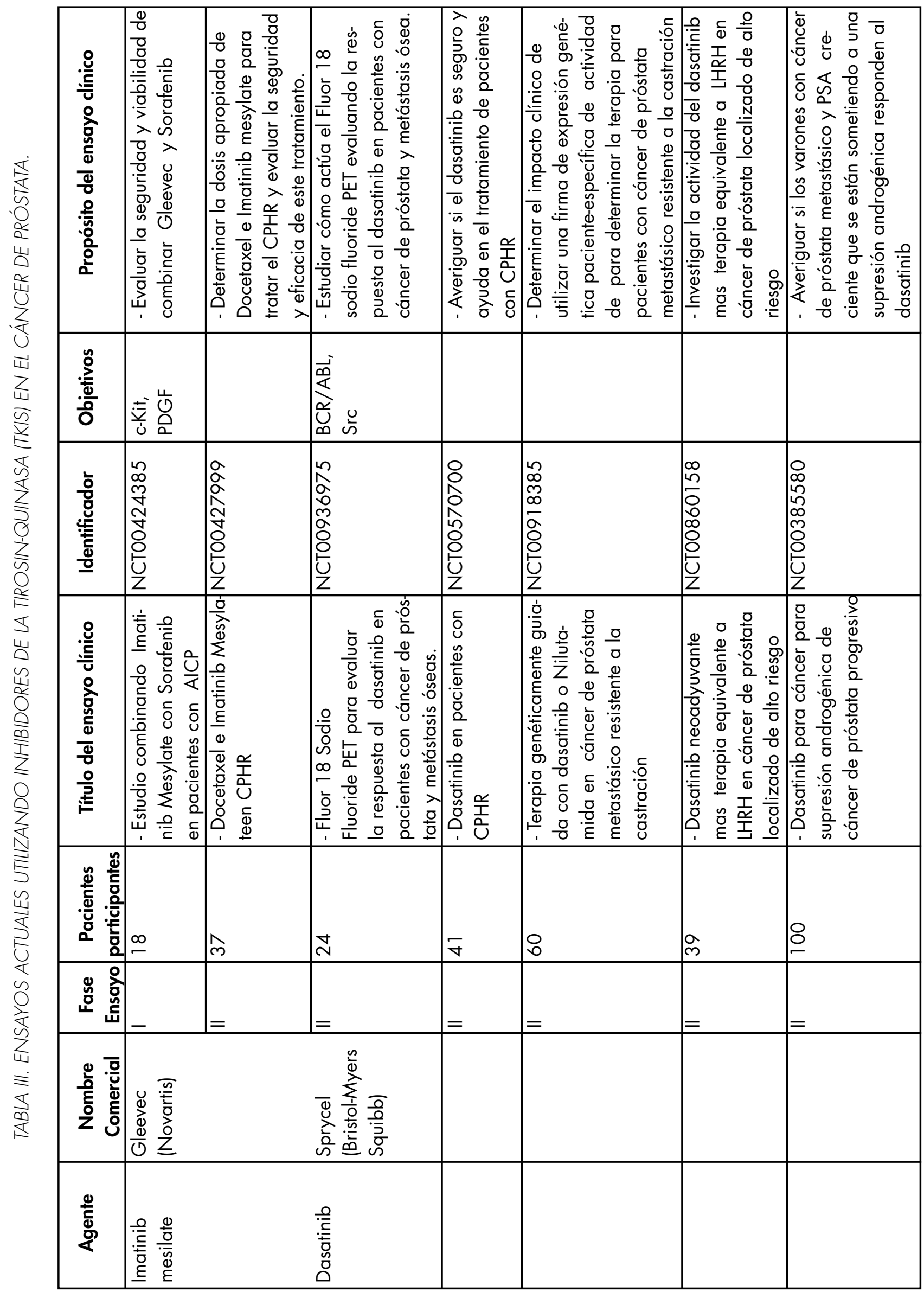









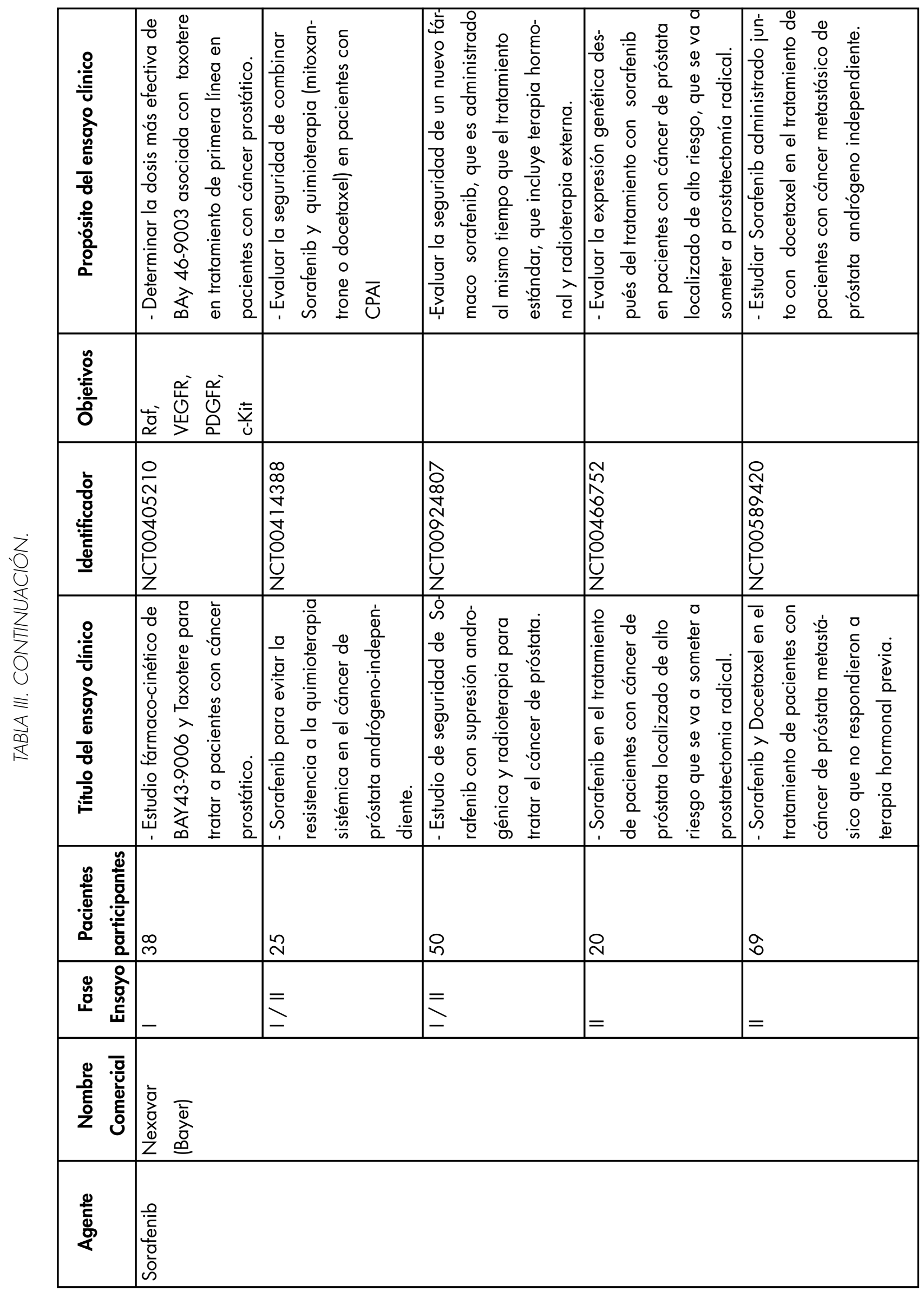


del $50 \%$ del PSA durante 2,5 meses y casi todos los pacientes tuvieron alivio del dolor necesitando menor medicación. Recientemente se han publicado dos ensayos de fase II investigando la actividad como agente único de gefitinib en el CPHR.

Canil et al investigaron la eficacia y toxicidad del gefitinib en un estudio multi-céntrico aleatorio. 47 Cuarenta pacientes con CPHR mínimamente sintomático fueron asignados aleatoriamente a 250 $\mathrm{mg} \circ 500 \mathrm{mg}$ diarios de gefitinib. Ninguno de los pacientes mostraron una disminución significativa de PSA u otro tipo de respuesta objetiva apreciable. Sin embargo 35 pacientes (87\%) lograron la estabilización de los niveles de PSA durante 2,5 a 16,5 meses. Small et al. estudiaron el efecto de $500 \mathrm{mg}$ al día de gefitinib en un estudio abierto multicéntrico. $48 \mathrm{De}$ los 40 pacientes evaluables con CPHR no metastásiCo, ninguno tuvo una disminución en los niveles de PSA > 50\% y sólo 3 tuvieron un PSA estable durante los 6 meses de tratamiento. El nivel de expresión del RFCE se determinó en 16 pacientes y la mayoría (12) mostraron tinción importante. No hubo relación entre la expresión de RFCE y la disminución del PSA. Tambien se estudió la calidad de vida y no mejoró de forma significativa cocon el tratamiento con gefitinib en este estudio.

El erlotinib (Tarceva ${ }^{\circledR}$, Roche) se dirige al RFCE (ErbB-1). Se introdujo por primera vez en la recurrencia del cáncer de pulmón no microcítico, y ha demostrado mejorar la supervivencia cuando se administra como agente único. Gravis et al, estudiaron el efecto del erlotinib en un ensayo monocéntrico de fase II (49). Se administró erlotinib 150 a 200 $\mathrm{mg}$ diarios hasta la progresión de la enfermedad a treinta pacientes con $\mathrm{CaP}$ avanzado o metastásico. El erlotinib mostró un beneficio clínico con una mejoría en el $40 \%$ de los pacientes en el indice de Karnofsky. Ningún paciente tuvo una disminución del PSA, pero el $14 \%$ logró la estabilización y el tiempo de duplicación del PSA se incrementó en un $33 \%$ de los pacientes. Gross et al, investigaron la asociación de erlotinib y docetaxel en un ensayo multicéntrico de fase II (50). Veintidós pacientes fueron tratados con docetaxel $60 \mathrm{mg} / \mathrm{m} 2$ en el día 1 y $150 \mathrm{mg}$ de erlotinib en los días 1 al 21 . Ocho pacientes no tuvieron respuesta objetiva, pero en 6 hubo una disminución > $50 \%$ en las cifras de PSA.

\section{FCEV en el cáncer de próstata}

El sorafenib (Nexavar ${ }^{\circledR}$, Bayer) es un inhibidor multi-quinasa orientado a la vía kinasa Ras/Raf, el FCEV y el factor de crecimiento derivado de las plaquetas.
En el ámbito del CPHR se han desarrollado numerosos estudios en fase II. El primer ensayo reclutó 22 pacientes con CPHR que recibieron 400 $\mathrm{mg}$ dos veces al día durante 28 días (51). Ninguno de ellos tuvo disminución $>50 \%$ de PSA, y 7 pacientes estuvieron libres de progresión de PSA en 4 meses $(31,8 \%)$. La mediana del tiempo hasta la progresión del PSA fue de 8 semanas (rango de 6 a $41)$. No obstante, los autores encontraron resultados discordantes entre las respuestas de PSA y evidencias radiográficas de metástasis óseas: dos pacientes tuvieron reducción en el nivel de PSA, pero evidencias radiográfica de progresión de metástasis. Además, de los pacientes que muestran progresión de la enfermedad después del primer ciclo de tratamiento $(n=$ 21), 13 han progresado sólo por criterios de PSA y 6 de ellos tenían una disminución espontánea del PSA después de que se suspendió el fármaco $(28,6 \%)$. El ensayo fue ampliado a 46 pacientes (52). Se modificaron los criterios de progresión de la enfermedad y la evidencia radiográfica de nuevas lesiones o progresiones metastásicas fueron valoradas según los «Criterios de evaluación de respuesta en Tumores Sólidos (RECIST)».

La progresión de PSA se ha valorado pero no utilizado como criterio de progresión. De los 24 pacientes que participaron en la segunda fase del estudio, 10 tuvieron enfermedad estable $(41,6 \%)$ y la duración media de la estabilización de la enfermedad fue de 18 semanas (rango de 15 a 48). Del mismo modo, Chi et al encontraron sólo en el 3,6\% disminución de PSA $>50 \%$ tras 4 semanas de tratamiento (53). Ambos ensayos sugieren que el PSA no es un marcador adecuado para la evaluación de la eficacia del sorafenib después de un ciclo de tratamiento.

Otro ensayo interesante con participación de 55 pacientes con CPHR no tratados previamente con quimioterapia recibieron $400 \mathrm{mg}$ de sorafenib dos veces al día continuamente durante 12 semanas (54). Quince pacientes mostraron estabilización de la enfermedad después de 12 semanas $(27,3 \%)$ y 2 pacientes presentaron disminución > 50\% de PSA $(3,6 \%)$. La mediana de tiempo libre de progresión de supervivencia fue de 8 semanas (rango 6,4 a 14,7). Entre los pacientes con enfermedad estable, 11 habían estabilizado los niveles de PSA después de 12 semanas de tratamiento.

\section{Otros TKIs en cáncer de próstata}

Las quinasas de la familia Src (SFKs) son la mayor familia de tirosin-quinasas no receptoras de proteínas y son responsables de una transducción de 
señal durante la diferenciación, la adhesión y migración de las células tumorales.

El dasatinib (Sprycel $\circledR_{\text {, Bristol-Myers Squibb) }}$ es una pequeña molécula inhibidora de tirosin-quinasa que bloquea SFKs, factor de crecimiento derivado de plaquetas, c-kit, Bcr-Abl y efrinas (55). Los estudios preclínicos demostraron que el dasatinib inhibe las actividades de las quinasas SFKs, Lyn y Src en ambos, CaP andrógeno-dependiente e independiente. Nam et al. descubrieron una correlación entre la inhibición de estas actividades de la quinasa por el dasatinib y la reducción de la adhesión, migración e invasión celular en sistemas de modelos in vitro (56). Los mismos efectos fueron observados por Park et al, en modelos en ratón (57). Se halló que el dasatinib inhibe el crecimiento de metástasis de los ganglios linfáticos del CaP. Recientemente se ha informado de los resultados preliminares de un estudio de fase II, incluyendo 27 pacientes con CPHR y metástasis óseas que fueron tratados con dasatinib durante 12 semanas consecutivas. 16 tuvieron enfermedad estable y 1 presentó una mejoría en las exploraciones radiológicas óseas después de 12 semanas de tratamiento.

\section{CONCLUSIÓN}

Los TKIs representan un tratamiento potencial prometedor del CPHR pero tienen que diseñarse más estudios fase III. Las terapias múltiples de bloqueo de quinasas, probablemente puedan ser más eficientes. Hasta ahora, se utilizan pocas terapias dirigidas en el cáncer de vejiga, pero parece evidente que los agentes quimioterápicos comunes deben ir acompañados por una nueva generación de fármacos que reconozcan objetivos específicos en o sobre las células tumorales. Este nuevo enfoque es muy prometedor en el tratamiento, en un futuro próximo de los tumores urológicos.

\section{BIBLIOGRAFÍA Y LECTURAS RECOMENDADAS (*lectura de interés $y$ ** lectura fundamental)}

1. Vojtek AB, Hollenberg SM and Cooper JA: Mammalian Ras interacts directly with the serine/ threonine kinase Raf. Cell. 74: 205-14, 1993.

2. Ciardiello F, Caputo R, Bianco R, Damiano V, Fontanini G, Cuccato S, De Placido S, Bianco AR and Tortora G: Inhibition of growth factor production and angiogenesis in human cancer cells by ZD1839 (Iressa), a selective epidermal growth factor receptor tyrosine kinase inhibitor. Clin Cancer Res. 7: 1459-65, 2001.

3. Stommel JM, Kimmelman AC, Ying H, Nabioullin R, Ponugoti AH, Wiedemeyer R, Stegh AH,
Bradner JE, Ligon KL, Brennan C et al.: Coactivation of receptor tyrosine kinases affects the response of tumor cells to targeted therapies. Science. 318: 287-90, 2007.

4. Motzer RJ, Hutson TE, Tomczak P, Michaelson MD, Bukowski RM, Rixe O, Oudard S, Negrier S, Szczylik C, Kim ST et al.: Sunitinib versus interferon alfa in metastatic renal-cell carcinoma. $\mathrm{N}$ Engl J Med. 356: 115-24, 2007.

5. Jemal A, Siegel R, Ward E, Hao Y, Xu J, Murray T and Thun MJ: Cancer statistics, 2008. CA Cancer J Clin. 58: 71-96, 2008.

6. Botteman MF, Pashos CL, Redaelli A, Laskin B and Hauser R: The health economics of bladder cancer: a comprehensive review of the published literature. Pharmacoeconomics. 21: 1315-30, 2003.

7. Baselga $\mathbf{J}$ and Averbuch SD: ZD1839 ('Iressa') as an anticancer agent. Drugs. 60 Suppl 1: 33-40; discussion 41-2, 2000.

8. Glading A, Chang P, Lauffenburger DA and Wells A: Epidermal growth factor receptor activation of calpain is required for fibroblast motility and occurs via an ERK/MAP kinase signaling pathway. J Biol Chem. 275: 2390-8, 2000.

9. Kassouf W, Black PC, Tuziak T, Bondaruk J, Lee S, Brown GA, Adam L, Wei C, Baggerly K, BarEli M et al.: Distinctive expression pattern of ErbB family receptors signifies an aggressive variant of bladder cancer. J Urol. 179: 353-8, 2008.

10. Junttila TT, Laato M, Vahlberg T, Soderstrom KO, Visakorpi $\mathrm{T}$, Isola $\mathrm{J}$ and Elenius $\mathrm{K}$ : Identification of patients with transitional cell carcinoma of the bladder overexpressing ErbB2, ErbB3, or specific ErbB4 isoforms: real-time reverse transcription-PCR analysis in estimation of ErbB receptor status from cancer patients. Clin Cancer Res. 9: 5346-57, 2003.

11. Huang S, Armstrong EA, Benavente S, Chinnaiyan P and Harari PM: Dual-agent molecular targeting of the epidermal growth factor receptor (EGFR): combining anti-EGFR antibody with tyrosine kinase inhibitor. Cancer Res. 64: 535562, 2004.

12. McHugh LA, Kriajevska M, Mellon JK and Griffiths TR: Combined treatment of bladder cancer cell lines with lapatinib and varying chemotherapy regimens--evidence of schedule-dependent synergy. Urology. 69: 390-4, 2007.

13. Natale RB: Biologically targeted treatment of non-small-cell lung cancer: focus on epidermal growth factor receptor. Clin Lung Cancer. 5 Suppl 1: S11-7, 2003.

14. Black PC, Brown GA, Inamoto T, Shrader M, Arora A, Siefker-Radtke AO, Adam L, Theodorescu D, Wu X, Munsell MF et al.: Sensitivity to epidermal growth factor receptor inhibitor requi- 
res E-cadherin expression in urothelial carcinoma cells. Clin Cancer Res. 14: 1478-86, 2008.

15. Philips GK, Halabi S, Sanford BL, Bajorin D and Small EJ: A phase II trial of cisplatin, fixed doserate gemcitabine and gefitinib for advanced urothelial tract carcinoma: results of the Cancer and Leukaemia Group B 90102. BJU Int. 101: 20-5, 2008.

16. Chow NH, Chan SH, Tzai TS, Ho CL and Liu HS: Expression profiles of ErbB family receptors and prognosis in primary transitional cell carcinoma of the urinary bladder. Clin Cancer Res. 7: 1957-62, 2001.

17. Kruger S, Weitsch G, Buttner H, Matthiensen A, Bohmer T, Marquardt T, Sayk F, Feller AC and Bohle A: HER2 overexpression in muscle-invasive urothelial carcinoma of the bladder: prognostic implications. Int J Cancer. 102: 514-8, 2002.

18. Bellmunt J, Hussain M and Dinney CP: Novel approaches with targeted therapies in bladder cancer. Therapy of bladder cancer by blockade of the epidermal growth factor receptor family. Crit Rev Oncol Hematol. 46 Suppl: S85-104, 2003.

19. Wulfing C, von Struensee D, Bierer S, Bogemann M, Hertle L and Eltze E: [Expression of Her2/neu in locally advanced bladder cancer: implication for a molecular targeted therapy]. Aktuelle Urol. 36: 423-9, 2005.

20. Crew JP: Vascular endothelial growth factor: an important angiogenic mediator in bladder cancer. Eur Urol. 35: 2-8, 1999.

21 Slaton JW, Millikan R, Inoue K, Karashima T, Czerniak B, Shen Y, Yang Y, Benedict WF and Dinney CP: Correlation of metastasis related gene expression and relapse-free survival in patients with locally advanced bladder cancer treated with cystectomy and chemotherapy. J Urol. 171: 5704, 2004.

22. Goddard JC, Sutton CD, Furness PN, O'Byrne $\mathrm{KJ}$ and Kockelbergh RC: Microvessel density at presentation predicts subsequent muscle invasion in superficial bladder cancer. Clin Cancer Res. 9: 2583-6, 2003.

23. Canoglu A, Gogus C, Beduk Y, Orhan D, Tulunay $\mathrm{O}$ and Baltaci S: Microvessel density as a prognostic marker in bladder carcinoma: correlation with tumor grade, stage and prognosis. Int Urol Nephrol. 36: 401-5, 2004.

24. Gallagher DJ and Bajorin DF: Neoadjuvant chemotherapy for the treatment of muscle-invasive bladder cancer: argument in favor. Nat Clin Pract Urol. 5: 484-5, 2008.

25. Sonpavde G, Ross R, Powles T, Sweeney CJ, Hahn N, Hutson TE, Galsky MD, Lerner SP and Sternberg CN: Novel agents for muscle-invasive and advanced urothelial cancer. BJU Int. 101: 937-43, 2008.

26. Omerovic J, Laude AJ and Prior IA: Ras proteins: paradigms for compartmentalised and isoform- specific signalling. Cell Mol Life Sci. 64: 257589, 2007.

27. Rosenberg JE, von der Maase H, Seigne JD, Mardiak J, Vaughn DJ, Moore M, Sahasrabudhe D, Palmer PA, Perez-Ruixo JJ and Small EJ: A phase II trial of R115777, an oral farnesyl transferase inhibitor, in patients with advanced urothelial tract transitional cell carcinoma. Cancer. 103: 2035-41, 2005.

28. Theodore C, Geoffrois L, Vermorken JB, Caponigro F, Fiedler W, Chollet P, Ravaud A, Peters GJ, de Balincourt C, Lacombe D et al.: Multicentre EORTC study 16997: feasibility and phase II trial of farnesyl transferase inhibitor \& gemcitabine combination in salvage treatment of advanced urothelial tract cancers. Eur J Cancer. 41: 1150-7, 2005.

29. Heidenreich A, Aus G, Bolla M, Joniau S, Matveev VB, Schmid HP and Zattoni F: EAU guidelines on prostate cancer. Eur Urol. 53: 68-80, 2008.

30. Wingo PA, Ries LA, Parker SL and Heath CW, Jr.: Long-term cancer patient survival in the United States. Cancer Epidemiol Biomarkers Prev. 7: 271-82, 1998.

31. Pilat MJ, Kamradt JM and Pienta KJ: Hormone resistance in prostate cancer. Cancer Metastasis Rev. 17: 373-81, 1998.

32. Tannock IF, de Wit R, Berry WR, Horti J, Pluzanska A, Chi KN, Oudard S, Theodore C, James ND, Turesson I et al.: Docetaxel plus prednisone or mitoxantrone plus prednisone for advanced prostate cancer. N Engl J Med. 351: 1502-12, 2004.

33. Petrylak DP, Tangen CM, Hussain MH, Lara PN, Jr., Jones JA, Taplin ME, Burch PA, Berry D, Moinpour C, Kohli M et al.: Docetaxel and estramustine compared with mitoxantrone and prednisone for advanced refractory prostate cancer. $\mathrm{N}$ Engl J Med. 351: 1513-20, 2004.

34. Edwards J, Krishna NS, Witton CJ and Bartlett JM: Gene amplifications associated with the development of hormone-resistant prostate cancer. Clin Cancer Res. 9: 5271-81, 2003.

35. Gioeli D, Mandell JW, Petroni GR, Frierson HF, Jr. and Weber MJ: Activation of mitogen-activated protein kinase associated with prostate cancer progression. Cancer Res. 59: 279-84, 1999.

36. Yarden Y: The EGFR family and its ligands in human cancer. signalling mechanisms and therapeutic opportunities. Eur J Cancer. 37 Suppl 4: S3-8, 2001.

37. Di Lorenzo G, Tortora G, D’Armiento FP, De Rosa G, Staibano S, Autorino R, D'Armiento M, De Laurentiis M, De Placido S, Catalano G et al.: Expression of epidermal growth factor receptor correlates with disease relapse and progression to androgen-independence in human prostate cancer. Clin Cancer Res. 8: 3438-44, 2002. 
38. Shah RB, Ghosh D and Elder JT: Epidermal growth factor receptor (ErbB1) expression in prostate cancer progression: correlation with androgen independence. Prostate. 66: 1437-44, 2006.

39. Osman I, Scher HI, Drobnjak M, Verbel D, Morris M, Agus D, Ross JS and Cordon-Cardo C: HER$2 /$ neu (p185neu) protein expression in the natural or treated history of prostate cancer. Clin Cancer Res. 7: 2643-7, 2001.

40. Scher HI, Sarkis A, Reuter V, Cohen D, Netto G, Petrylak D, Lianes P, Fuks Z, Mendelsohn J and Cordon-Cardo C: Changing pattern of expression of the epidermal growth factor receptor and transforming growth factor alpha in the progression of prostatic neoplasms. Clin Cancer Res. 1: 545-50, 1995.

41. Hanahan D and Folkman J: Patterns and emerging mechanisms of the angiogenic switch during tumorigenesis. Cell. 86: 353-64, 1996.

42. Mimeault M, Pommery N and Henichart JP: New advances on prostate carcinogenesis and therapies: involvement of EGF-EGFR transduction system. Growth Factors. 21: 1-14, 2003.

43. Sirotnak FM, Zakowski MF, Miller VA, Scher HI and Kris MG: Efficacy of cytotoxic agents against human tumor xenografts is markedly enhanced by coadministration of ZD1839 (Iressa), an inhibitor of EGFR tyrosine kinase. Clin Cancer Res. 6: 4885-92, 2000.

44. Baselga J, Rischin D, Ranson M, Calvert H, Raymond E, Kieback DG, Kaye SB, Gianni L, Harris A, Bjork T et al.: Phase I safety, pharmacokinetic, and pharmacodynamic trial of ZD1839, a selective oral epidermal growth factor receptor tyrosine kinase inhibitor, in patients with five selected solid tumor types. J Clin Oncol. 20: 4292-302, 2002.

45. Ranson M, Hammond LA, Ferry D, Kris M, Tullo A, Murray PI, Miller V, Averbuch S, Ochs J, Morris $\mathrm{C}$ et al.: ZD1839, a selective oral epidermal growth factor receptor-tyrosine kinase inhibitor, is well tolerated and active in patients with solid, malignant tumors: results of a phase I trial. J Clin Oncol. 20: 2240-50, 2002.

46. Herbst RS, Maddox AM, Rothenberg ML, Small EJ, Rubin EH, Baselga J, Rojo F, Hong WK, Swaisland H, Averbuch SD et al.: Selective oral epidermal growth factor receptor tyrosine kinase inhibitor ZD1839 is generally well-tolerated and has activity in non-small-cell lung cancer and other solid tumors: results of a phase I trial. J Clin Oncol. 20: 3815-25, 2002.

47. Canil CM, Moore MJ, Winquist E, Baetz T, Pollak M, Chi KN, Berry S, Ernst DS, Douglas L, Brundage $\mathrm{M}$ et al.: Randomized phase II study of two doses of gefitinib in hormone-refractory prostate cancer: a trial of the National Cancer Institute of Canada-Clinical Trials Group. J Clin Oncol. 23: 455-60, 2005.
48. Small EJ, Fontana J, Tannir N, DiPaola RS, Wilding G, Rubin M, Iacona RB and Kabbinavar FF: A phase II trial of gefitinib in patients with nonmetastatic hormone-refractory prostate cancer. BJU Int. 100: 765-9, 2007.

49. Gravis G, Bladou F, Salem N, Goncalves A, Esterni B, Walz J, Bagattini S, Marcy M, Brunelle S and Viens P: Results from a monocentric phase II trial of erlotinib in patients with metastatic prostate cancer. Ann Oncol. 19: 1624-8, 2008.

50. Gross M, Higano C, Pantuck A, Castellanos O, Green E, Nguyen K and Agus DB: A phase II trial of docetaxel and erlotinib as first-line therapy for elderly patients with androgen-independent prostate cancer. BMC Cancer. 7: 142, 2007.

51. Dahut WL, Scripture C, Posadas E, Jain L, Gulley JL, Arlen PM, Wright JJ, Yu Y, Cao L, Steinberg SM et al.: A phase II clinical trial of sorafenib in androgen-independent prostate cancer. Clin Cancer Res. 14: 209-14, 2008.

52. Aragon-Ching JB, Jain L, Gulley JL, Arlen PM, Wright JJ, Steinberg SM, Draper D, Venitz J, Jones E, Chen CC et al.: Final analysis of a phase II trial using sorafenib for metastatic castration-resistant prostate cancer. BJU Int. 103: 1636-40, 2009.

53. Chi KN, Ellard SL, Hotte SJ, Czaykowski P, Moore M, Ruether JD, Schell AJ, Taylor S, Hansen C, Gauthier I et al.: A phase II study of sorafenib in patients with chemo-naive castration-resistant prostate cancer. Ann Oncol. 19: 746-51, 2008.

54. Steinbild S, Mross K, Frost A, Morant R, Gillessen S, Dittrich C, Strumberg D, Hochhaus A, Hanauske AR, Edler L et al.: A clinical phase II study with sorafenib in patients with progressive hormonerefractory prostate cancer: a study of the CESAR Central European Society for Anticancer Drug Research-EWIV. Br J Cancer. 97: 1480-5, 2007.

55. Lombardo LJ, Lee FY, Chen P, Norris D, Barrish JC, Behnia K, Castaneda S, Cornelius LA, Das J, Doweyko AM et al.: Discovery of N-(2chloro-6-methyl- phenyl)-2-(6-(4-(2-hydroxyethyl)- piperazin-1-yl)-2-methylpyrimidin-4ylamino)thiazole-5-carboxamide (BMS-354825), a dual Src/Abl kinase inhibitor with potent antitumor activity in preclinical assays. J Med Chem. 47: 6658-61, 2004.

56. Nam S, Kim D, Cheng JQ, Zhang S, Lee JH, Buettner R, Mirosevich J, Lee FY and Jove R: Action of the Src family kinase inhibitor, dasatinib (BMS-354825), on human prostate cancer cells. Cancer Res. 65: 9185-9, 2005.

57. Park SI, Zhang J, Phillips KA, Araujo JC, Najjar AM, Volgin AY, Gelovani JG, Kim SJ, Wang Z and Gallick GE: Targeting SRC family kinases inhibits growth and lymph node metastases of prostate cancer in an orthotopic nude mouse model. Cancer Res. 68: 3323-33, 2008. 